

\title{
Turbulent wake past a three-dimensional blunt body. Part 2. Experimental sensitivity analysis
}

\author{
Mathieu Grandemange, Marc Gohlke, Olivier Cadot
}

\section{To cite this version:}

Mathieu Grandemange, Marc Gohlke, Olivier Cadot. Turbulent wake past a three-dimensional blunt body. Part 2. Experimental sensitivity analysis. Journal of Fluid Mechanics, 2014, 752, pp.439-461. hal-01161541

\section{HAL Id: hal-01161541 \\ https://hal.science/hal-01161541}

Submitted on 8 Jun 2015

HAL is a multi-disciplinary open access archive for the deposit and dissemination of scientific research documents, whether they are published or not. The documents may come from teaching and research institutions in France or abroad, or from public or private research centers.
L'archive ouverte pluridisciplinaire HAL, est destinée au dépôt et à la diffusion de documents scientifiques de niveau recherche, publiés ou non, émanant des établissements d'enseignement et de recherche français ou étrangers, des laboratoires publics ou privés. 


\title{
Turbulent wake past a three-dimensional blunt body. Part 2. Experimental sensitivity analysis.
}

\author{
M. GRA N D EMA N G E $\mathbf{H}^{1,2}$, M. G OHL K E \\ AND O. CADOT T \\ ${ }^{1}$ Unité de Mécanique, Ecole Nationale Supérieure de Techniques Avancées, ParisTech, \\ 828 Boulevard des Maréchaux, 91762 Palaiseau Cedex, France \\ ${ }^{2}$ PSA Peugeot Citroën, Centre Technique de Vélizy, \\ Route de Gisy, 78943 Vélizy-Villacoublay Cedex, France
}

(Received ?; revised ?; accepted ?. - To be entered by editorial office)

The sensitivity of the flow around the three-dimensional blunt geometry is investigated experimentally at Reynolds number $9.210^{4}$. Vertical and horizontal control cylinders are used to disturb the natural flow which is the superposition of two reflectional symmetry breaking states (Grandemange et al. 2013b). When the perturbation breaks the symmetry of the set-up, it can select one of the two asymmetric topologies so that a mean side force is reported. When the reflectional symmetry is preserved, some positions of horizontal and vertical control cylinders alter the natural bi-stability of the flow which may result in drag reduction. In addition, it is found that the horizontal perturbation affects the lift force especially when the top and bottom mixing layers are disturbed. The ability of the disturbances to suppress the bistable behavior is discussed and, introducing a formalism of induced drag, a quantification of the impact on the drag of the cross-flow forces observed for the natural bistable wake is suggested. Finally, a general concept for control strategy of separated flows past three-dimensional bluff bodies can be drawn from these analyses.

\section{Introduction}

In the transport industry, economical and environmental issues on energy consumption are motivating research activities to limit the drag of road vehicles. The objective is to develop efficient strategies of shape optimization or flow control. Due to their functional shapes, the geometries are bluff from an aerodynamic point of view and the flow is separated on the after-body. In order to achieve the drag reductions needed by industry, the comprehension of such flows should be investigated for simplified three-dimensional geometries.

An important work is devoted to the axisymmetric configurations (spheres, disks and bullet shaped bodies) for which steady and unsteady global modes are evidenced, both experimentally (Magarvey \& Bishop 1961; Sakamoto \& Haniu 1990; Ormières \& Provansal 1999; Siegel et al. 2008; Szaltys et al. 2012) and numerically (Fabre et al. 2008; Pier 2008; Meliga et al. 2009a). When the Reynolds number increases in the laminar regime, a pitchfork bifurcation leads to a steady flow that breaks the axisymmetry; then, after a second bifurcation, an unsteady antisymmetric mode associated with the shedding of vortex loops appears in the wake. The numerical results of Pier (2008) highlight the impact of these symmetry breaking modes on the fluid forces: after the axisymmetry 
breaking of the sphere wake at Reynolds number 210, he reports the emergence of a side force and of an additional drag which is responsible for approximately $10 \%$ of the total drag at $\operatorname{Re}=350$.

In parallel, theoretical developments allow the structural sensitivity of global mode wakes to be obtained in the laminar regime (Hill 1992; Marquet et al. 2008; Luchini et al. 2009). This approach is able to recover qualitatively the experimental sensitivity maps of Strykowski \& Sreenivasan (1990) over bi-dimensional geometries. Extending the method to the turbulent flow around a "D" shaped cylinder at Re $=1.310^{4}$, Meliga et al. (2012) present a sensitivity map of the global mode frequency that is in good agreements with the experimental results of Parezanovic \& Cadot (2012), both in terms of spatial distribution and amplitude. The modifications induced by the perturbation are related to the introduction of a stagnation point in the main wake. This generates vorticity in the flow, whose interaction with the main flow vorticity is at the origin of the sensitivity (Parezanović \& Cadot 2009). It appears that the particular feature of the vorticity, i.e. the possible presence of vortex shedding downstream of the disturbance, is not relevant; for example, Meliga et al. (2012) model the perturbation by a steady local forcing in the main wake. This approach appears as a useful tool in the framework of flow control since it predicts where a disturbance efficiently affects global properties such as drag or global mode frequency. Thus, for practical and industrial interests, the method should be extended to larger Reynolds number flows over more complex geometries. The identification of the sensitive regions in the flow should help to define efficient strategies of drag reduction that could be performed, in a second step, using realistic control devices such as surface mounted actuators.

Previous control experiments of axisymmetric turbulent wakes address the development of the unsteady modes, showing connections between the control parameters, the activity of the mode and the drag (Berger et al. 1990; Weickgenannt \& Monkewitz 2000; Sevilla \& Martinez-Bazan 2004; Higuchi 2005; Morrison \& Qubain 2009). However, in most of these cases, the control techniques cannot be considered as perturbations. Now, by placing small control obstacles downstream of axisymmetric bodies, some experiments explore the sensitivity of the flow to steady local disturbances. First, one can refer to the work of Grandemange et al. $(2012 b)$ who consider the case of an axisymmetric bullet shaped body; due to the $m=2$ azimuthal periodicity of the support, the wake presents two preferred orientations leading to a bistable flow. The results prove that the coexistence of the two asymmetric states is strongly affected by any antisymmetric perturbation. Moreover, these experiments confirm the sensitivity of the center of the recirculation bubble in which the bi-stability is suppressed together with a drag reduction. In this region, the perturbation seems to control the reminiscences of the steady axisymmetry breaking mode whose theoretical sensitivity is given in Meliga et al. (2009b) for a sphere and a disk.

Similar bistable wakes can equally be reported for flows past more complex geometries with only one reflectional plane of symmetry. It has been observed in the separated flows over the rear window of a notch-back vehicle (Lawson et al. 2007) or past a threedimensional double backward facing step (Herry et al. 2011). High degrees of asymmetry have also been obtained in experiments and numerical simulations of the flow past threedimensional geometries despite the symmetry of the set-up (Lee et al. 2009; Wassen et al. 2010); they might be the signature of a comparable instability. Analogous dynamics in the natural wake past the reference Ahmed geometry (Ahmed et al. 1984) are also reported in Grandemange et al. (2013b). In that particular case, the wake presents two different time scales. 
- At short timescales $T_{s} \sim 5 H / U_{0}$, weak coherent oscillations in the vertical and lateral directions are reported; they are associated with the interaction of the facing shear layers.

- At long timescales $T_{l} \sim 10^{3} \mathrm{H} / U_{0}$, the recirculation region shifts between two preferred reflectional symmetry breaking positions, the equiprobability of the two states leading to a statistical symmetric wake. The succession of these two asymmetric states is random and results in an unsteady side force that overall average is zero. This phenomenon is independent of the Reynolds number; it is ascribed to the emergence of a steady reflectional symmetry breaking mode in the laminar regime (Grandemange et al. 2012a). Indeed, at $\operatorname{Re}=U_{0} H / \nu=340 \pm 10, H$ being the height of the base, the wake undergoes a pitchfork bifurcation from a trivial steady symmetric state to a steady asymmetric regime. The generality of such cross-flow instabilities is proved by the wide domains of appearance identified in Grandemange et al. (2013a).

In this framework, the present study explores the sensitivity of the flow past the squareback model used in Ahmed et al. (1984). These experiments complete the analysis of the natural wake provided in Grandemange et al. (2013b). The objective is to clarify how a local disturbance affects the global properties of the flow with a particular focus on its bistable characteristic.

The article is organized as follows. In section 2, the experimental set-up and measurements are presented. Section 3 is devoted to the results: analysis of the effects of vertical and horizontal control cylinders. The ability to suppress the bi-stability as well as the link between the drag and the cross-flow forces are discussed in section 4. Finally, concluding remarks are presented in section 5 .

\section{Experimental set-up}

The experimental facilities are identical to Grandemange et al. (2013b). The geometry and the disturbance set-up are successively presented and the measurements of pressure, force and velocity are detailed.

\subsection{Geometry}

The experimental set-up is illustrated in figure 1. A ground plate is placed in an Eiffel type wind tunnel to form a $3 / 4$ open jet facility (open test section on the sides and on the top). The turbulent intensity is less than $0.3 \%$ and the homogeneity of the velocity over the $390 \mathrm{~mm} \times 400 \mathrm{~mm}$ section is $0.4 \%$. The wake is generated by the square-back geometry used in the experiments of Ahmed et al. (1984) at scale 1/4. The total length of the body is $L=261.0 \mathrm{~mm}$, the height $H$ and width $W$ of the base are respectively $72.0 \mathrm{~mm}$ and $97.2 \mathrm{~mm}$. The four supports are cylindrical with a diameter of $7.5 \mathrm{~mm}$ and the ground clearance is $C=12.5 \mathrm{~mm}$ to match the reference experiments. The blockage ratio is less than $5 \%$. The coordinate system is defined as $x$ in the streamwise direction, $z$ normal to the ground and $y$ forming a direct trihedral.

In order to have constant flow conditions, the ground plate is placed at $10 \mathrm{~mm}$ above the bottom side of the inlet (see figure 1a) and triggers the turbulent boundary layer, without any separation, $140 \mathrm{~mm}$ upstream of the fore-body. When the body is not in the test section, the thickness of the ground boundary layer based on $99 \%$ of the free-flow velocity at $x=-L$, i.e. $140 \mathrm{~mm}$ downstream of the leading edge, is $\delta_{0.99}=6.3 \mathrm{~mm}$ with a precision of $0.1 \mathrm{~mm}$; the displacement and momentum thicknesses are $0.89 \pm 0.05 \mathrm{~mm}$ and $0.60 \pm 0.02 \mathrm{~mm}$ respectively. 
FiguRE 1. Experimental set-up: side view $(a)$, top view $(b)$ and perspective view $(c, d)$ disturbed by a vertical control cylinder $(c)$ and an horizontal control cylinder $(a, b, d)$; gray dots are pressure taps, they are distributed symmetrically referring to the planes $y^{*}=0$ and $z^{*}=0.67$ (mid-height of the body); $O$ sets the origin of the coordinate system.

The main flow velocity is $U_{0}=20 \mathrm{~m} \mathrm{~s}^{-1}$ and the Reynolds number is $\operatorname{Re}=U_{0} H / \nu=$ $9.210^{4}$. The velocities are defined as $\vec{u}=u_{x} \cdot \overrightarrow{e_{x}}+u_{y} \cdot \overrightarrow{e_{y}}+u_{z} \cdot \overrightarrow{e_{z}} ; u_{i j}=\sqrt{u_{i}{ }^{2}+u_{j}{ }^{2}}$ is the amplitude of velocity at the considered point in the plane $\left(\overrightarrow{e_{i}}, \overrightarrow{e_{j}}\right) . A($ or $\langle a\rangle)$ and $\operatorname{Std}(a)$ are respectively the average value and the standard deviation of any quantity $a$; $a^{\prime}=a-A$ is the fluctuating part of $a$. In the following, $a_{n}$ stands for the natural value of $a$ (characterized in Grandemange et al. 2013b). The height $H$ of the base, density $\rho$ and inlet velocity $U_{0}$ are used to obtain non-dimensional values marked with an asterisk.

\subsection{Disturbance apparatus for the sensitivity analysis}

The sensitivity analysis presented in the following is based on the experimental work of Parezanović \& Cadot (2012) in a bi-dimensional configuration. In this previous study, the flow is found sensitive when the control cylinder disturbs the shear layers. Given the parallelepiped shape of the present geometry and the shear layer interactions reported in the natural wake (Grandemange et al. 2013b), the direct extension of the work of Parezanović \& Cadot (2012) consists in disturbing the lateral mixing layers or the top and bottom ones. Hence, it appears suitable to use small vertical and horizontal control cylinders placed around the after-body as displayed in figure 1 . Their lengths are logically equal to the height or the width of the body (see figures $1 c, d$ ).

Besides, in Parezanović \& Cadot (2012), two different rod sizes are used; they correspond to $0.04 D$ and $0.12 D$ which should be compared to the initial thickness of the shear layer of $0.05 D, D$ being the main cylinder diameter. It is found that the smaller control cylinder can be considered as a disturbance while the larger one induces important modifications of the main flow features. Thus, the perturbation assumption remains relevant as soon as the control rod is smaller than the disturbed shears. In the present configuration, the diameter of the cylinders is $d=4 \mathrm{~mm}$, i.e. $d / W=4.1 \%$ and $d / H=5.5 \%$. This cylinder diameter corresponds to a compromise between the perturbation assumption and the amplitude of the measurable effect on the flow. As the initial thickness of the shear is of order of $0.03 H$ (Grandemange et al. 2013b), the cylinders may be too big to be considered as local disturbance in the very near wake. Nevertheless, as soon as $x>0.2 H$, the shear layers of the natural flow are larger than $0.05 H$ and the perturbation assumption becomes sustainable.

The vertical rod is moved in the $x$ and $y$ directions whereas the horizontal one is moved in the $x$ and $z$ directions; their positions are denoted by $\left(x_{C}, y_{C}\right)$ and $\left(x_{C}, z_{C}\right)$ respectively. The disturbance location is controlled by a three-dimensional displacement system made up of three Newport (M-)MTM long travel consoles and controlled by the Newport Motion Controller ESP301; the precision of the robots is better than $0.1 \mathrm{~mm}$. The disturbances are automatically displaced over a matrix by steps of $\Delta_{x}=3 \mathrm{~mm}$ in the streamwise direction and of $\Delta_{y}=\Delta_{z}=1 \mathrm{~mm}$ in the cross-flow directions, i.e. $\Delta_{x}{ }^{*}=$ 0.042 and $\Delta_{y}{ }^{*}=\Delta_{z}{ }^{*}=0.014$. The explored domains are $\left(x_{C}{ }^{*}, y_{C}{ }^{*}\right) \in[0.21,1.67] \times$ $[-0.97,0.97]$ and $\left(x_{C}{ }^{*}, z_{C}{ }^{*}\right) \in[0.21,1.67] \times[0.15,1.30]$. As a result, the full exploration of the wake by one disturbance typically needs more than 100 positions in the cross-flow direction and 35 in the $x$ direction.

The cylinders are held by $0.5 \mathrm{~mm}$ steel wires oriented along $y$ (one wire for the horizontal cylinder and two wires for the vertical one) in such a way that they are aligned with the base of the body. The supporting wires are sufficiently stretched to have vibra- 
tion amplitudes that are negligible compared to $d$. Additional tests with only the $0.5 \mathrm{~mm}$ wires have been performed to verify that their influence is at least one order of magnitude smaller that the one of the main control cylinders.

The construction of sensitivity maps are based on global quantities of the flow. Before detailing their measurements in sections 2.3 and 2.4, it is crucial to distinguish two different approaches in this work.

- First, the construction of the sensitivity maps corresponds to a statistical approach. They are obtained after the systematic displacement of the disturbance in the wake and, for each position, the data is recorded for $60 \mathrm{~s}$. This is too short to obtain converged values of mean and standard deviation, especially considering the long time dynamics of the bi-stability. These $60 \mathrm{~s}$ are limited by the total duration of an experiment which already lasts for two days. As a result, these sensitivity maps are only relevant as a whole and the accuracy of the associated data is visible through the dispersion of points in the scatter plots presented in the following.

- In addition, some specially control cylinder positions are studied in details in sections 3.1. They are associated with converged measurements, performed over $500 \mathrm{~s}$. The accuracy of the data is identical to the natural case detailed in Grandemange et al. $(2013 b)$.

\subsection{Pressure measurements}

The pressure on the body is measured at 62 locations (see gray dots in figures $1 c, d$ ). The taps are distributed symmetrically referring to the planes $y^{*}=0$ and $z^{*}=0.67$, the latter plane corresponding to the mid-height of the geometry. 21 taps are located on the base of the body; 41 others give the pressure distribution on the forebody and on the sides in the planes $y^{*}=0$ and $z^{*}=0.67$. The pressure is obtained using a 64 port HD miniature pressure scanner and a SCANdaq 8000 interface connected to a PC with Labview software. The scanner measures the pressure at $1 \mathrm{~Hz}$, as an average over the considered second, with an accuracy better than $3 \mathrm{~Pa}$. Hence, these measurements only catch the long time dynamics of the signal, at time scales larger that $1 \mathrm{~s}$. They are denoted $c_{p}$ in opposition to the mean pressure coefficient $C_{p}=\left\langle c_{p}\right\rangle$; similarly, $\operatorname{Std}\left(c_{p}\right)$ corresponds to the standard deviation of the $1 \mathrm{~Hz}$ measurement.

The pressure scanner is located inside the model; it is denoted " $P$ scan" in figures $1(a, b)$. It is linked to each tap with less than $250 \mathrm{~mm}$ of vinyl tube to limit the filtering effect of the tubing. This device is connected to the measurement chain using a wire going through a front support of the model so that, apart from the supports, nothing disturbs the underbody flow.

\subsection{Force measurements}

The dimensionless coefficient $c_{i}$ of the aerodynamic force $f_{i}$ in the $i$ direction is defined according to equation (2.1) using $S=7.1910^{-3} \mathrm{~m}^{2}$, the projected area of the geometry in a cross-flow plane.

$$
c_{i}=\frac{f_{i}}{\frac{1}{2} \rho S U_{0}^{2}},
$$

with $i \in\{x, y, z\}$.

The pressure contribution to the aerodynamic force in the $i$ direction, denoted $c_{i p}$, is estimated by integration of the pressure projected in the considered direction as defined in equation $(2.2)$.

$$
c_{i p}=\frac{1}{S} \int_{b o d y} c_{p} \overrightarrow{e_{i}} \cdot \overrightarrow{d s}
$$


with $c_{p}=\frac{p-p_{0}}{\frac{1}{2} \rho U_{0}^{2}}$ and $i \in\{x, y, z\}$.

Unlike the experiments of Grandemange et al. (2013b), the balance measurements are not used. As the sensitivity analyses need a continuous blowing of the wind tunnel for two days, there is an important daily drift of the balance signal, essentially due to temperature variations. Moreover, preliminary tests have shown that the balance-based and pressurebased sensitivity maps present identical trends: they contain the same information. As a result, the forces are estimated from the pressure measurements only. Using the formalism presented in section $2.3, c_{i p}$ refers to the force measurement at $1 \mathrm{~Hz}$, in opposition to $C_{i p}=\left\langle c_{i p}\right\rangle$ which is the time averaged pressure force.

\subsection{Particle image velocimetry}

Finally, wake analyses are made from particle image velocimetry (PIV). The system is comprised of a DANTEC dual pulse laser (Nd:YAG, $2 \times 135 \mathrm{~mJ}, 4 \mathrm{~ns}$ ) and a DANTEC CCD camera (FlowSense EO, $4 \mathrm{Mpx}$ ). The set-up acquires image pairs at a rate of $2 \mathrm{~Hz}$; each acquisition records 500 image pairs. The size of the interrogation window is $32 \times 32$ pixels with an overlap of $25 \%$. The measurements are performed in planes $y^{*}=0$ and $z^{*}=0.6$. The $32 \times 32$ pixels of the interrogation window correspond to physical sizes of $1.5 \times 1.5 \mathrm{~mm}$ in the plane $y^{*}=0$ and of $1.8 \times 1.8 \mathrm{~mm}$ in the plane $z^{*}=0.6$. The mean velocities are estimated from the valid vectors of the instantaneous velocity fields; these statistics are taken into account only when more than $50 \%$ of valid vectors are obtained from the 500 measurements.

From these velocity fields, the length of the recirculation region $L_{r}$ is obtained as the maximal streamwise position of the dividing streamlines of the recirculation bubble. Note that the plane at $z^{*}=0.6$ correspond to the $z$ coordinate of the closure of the natural recirculation bubble (Grandemange et al. 2013b). Hence, both planes at $y^{*}=0$ and $z^{*}=0.6$ allow the measurement of $L_{r}$.

\section{Results}

As presented in Grandemange et al. (2013b), the natural wake presents two preferred reflectional symmetry breaking positions; each asymmetric state is associated with a diagonal recirculating flow and a base pressure gradient in the $y$ direction: $\partial C_{p} / \partial y^{*}= \pm 0.17$. The states are denoted \# $\mathrm{P}$ and \# $\mathrm{N}$ for the positive and negative gradients respectively. The succession of the states is random and the equiprobability of the two asymmetric topologies leads to a symmetric mean flow; the bi-stability is characterized by the presence of two maxima in the probability distribution of $\partial c_{p} / \partial y^{*}$, centered on \pm 0.17 .

\subsection{Disturbed wake}

The disturbed flow for four typical cases of the vertical and horizontal control cylinder positions are first investigated. The analysis of these configurations is performed through PIV and pressure measurements. The corresponding velocity fields and statistics of $\partial c_{p} / \partial y^{*}$ are given in figure 2 (resp. figure 3) while the relevant quantities are listed in table 1 (resp. table 2) for the vertical control cylinder (resp. horizontal control cylinder).

The equiprobability of the states \#P and \#N relies on the symmetry of the set-up. As soon as the vertical control cylinder is out of the plane of symmetry, i.e. $y_{C}{ }^{*} \neq 0$, there is no reason for the flow to be symmetric. Figures $2(a, b)$ present the mean velocities in the plane $z^{*}=0.6$ and the base pressure statistics for the cylinder positions $\mathrm{V}_{a}$ and $\mathrm{V}_{b}$. For the position $\mathrm{V}_{a}$, the state \#N of the flow is selected (see figure $2 a$ ): the wake is clearly 
FiguRE 2. Velocity fields in the plane $z^{*}=0.6$ (left) and probability distributions of the base pressure gradient in the $y$ direction (right) for different positions of the vertical control cylinder: $\mathrm{V}_{a}$ for $x_{C}{ }^{*}=0.29$ and $y_{C}{ }^{*}=-0.60,(a) ; \mathrm{V}_{b}$ for $x_{C}{ }^{*}=0.79$ and $y_{C}{ }^{*}=-0.40,(b) ; \mathrm{V}_{c}$ for $x_{C}{ }^{*}=0.71$ and $y_{C}{ }^{*}=0,(c) ; \mathrm{V}_{d}$ for $x_{C}{ }^{*}=1.37$ and $y_{C}{ }^{*}=0,(d)$. Pressure measurements are performed over $500 \mathrm{~s}$ to obtain converged statistics. In the PIV, the missing data correspond to cylinder shadows and laser reflections; crosses are saddle points.

\begin{tabular}{|c|c|c|c|c|c|c|c|c|c|}
\hline Pos. & $x_{C}{ }^{*}$ & $y_{C}{ }^{*}$ & $\frac{\partial C_{p}}{\partial y^{*}}$ & $\operatorname{Std}\left(\frac{\partial c_{p}}{\partial y^{*}}\right)$ & $C_{x p}$ & $C_{y p}$ & $C_{z p}$ & $C_{p b}$ & $L_{r}{ }^{*}$ \\
\hline \multicolumn{2}{|c|}{ Natural $\ddagger$} & & 0.01 & 0.15 & 0.206 & 0.00 & -0.08 & -0.185 & 1.47 \\
\hline $\mathrm{V}_{a}$ & 0.29 & -0.60 & -0.15 & 0.03 & 0.206 & 0.02 & -0.08 & -0.188 & 1.52 \\
\hline $\mathrm{V}_{b}$ & 0.79 & -0.40 & 0.16 & 0.02 & 0.205 & -0.02 & -0.09 & -0.186 & 1.53 \\
\hline $\mathrm{V}_{c}$ & 0.71 & 0 & -0.02 & 0.08 & 0.194 & 0.00 & -0.08 & -0.175 & 1.57 \\
\hline \multirow[t]{2}{*}{$\mathrm{V}_{d}$} & 1.37 & 0 & -0.01 & 0.15 & 0.205 & 0.00 & -0.09 & -0.186 & 1.46 \\
\hline & & $\mathrm{cac}$ & \pm 0.01 & \pm 0.01 & 0.005 & \pm 0.01 & \pm 0.01 & \pm 0.003 & \pm 0.01 \\
\hline
\end{tabular}

TABLE 1. Global properties of the natural and disturbed wakes for four different positions of vertical control cylinder. †Data from Grandemange et al. (2013b).

FiguRE 3. Velocity fields in the plane $y^{*}=0$ (left) and probability distributions of the base pressure gradient in the $y$ direction (right) for different positions of the horizontal control cylinder: $\mathrm{H}_{a}$ for $x_{C}{ }^{*}=0.37$ and $z_{C}{ }^{*}=1.17,(a) ; \mathrm{H}_{b}$ for $x_{C}{ }^{*}=0.58$ and $z_{C}{ }^{*}=0.25,(b) ; \mathrm{H}_{c}$ for $x_{C}{ }^{*}=0.71$ and $z_{C}{ }^{*}=0.21,(c) ; \mathrm{H}_{d}$ for $x_{C}{ }^{*}=1.00$ and $z_{C}{ }^{*}=0.60,(d)$. Pressure measurements are performed over $500 \mathrm{~s}$ to obtain converged statistics. In the PIV, the missing data correspond to cylinder shadows and laser reflections; crosses are saddle points.

oriented toward the domain $y^{*}<0$; the mean diagonal recirculating flow corresponds to the presence of a single peak in the probability distribution of the base pressure gradient $\partial c_{p} / \partial y^{*}$ centered on $-0.16 \pm 0.01$. An almost mirror image of figure $2(a)$ is produced in figure $2(b)$ which shows that the state \#P is dominant for the position $\mathrm{V}_{b}$ : the wake is oriented toward the domain $y^{*}>0$, the diagonal recirculating flow is associated with the maximum in the probability distribution of $\partial c_{p} / \partial y^{*}$ centered on $0.16 \pm 0.01$. As a result, when $y_{C}{ }^{*} \neq 0$, the vertical disturbance can select one asymmetric state; the selected state depends not only on the sign of $y_{C}{ }^{*}$ but also on the streamwise position $x_{C}{ }^{*}$ of the disturbance. It can be seen that the base pressure and the pressure drag measured for these cylinder positions are very similar to the natural case indicating that there is no drag evolution (see table 1): the main effect of the disturbance is the selection of one asymmetric state.

The two other positions of the vertical control cylinder in figures $2(c, d)$ preserve the reflectional symmetry of the set-up and so, the observed flow is symmetric. For $\mathrm{V}_{c}$, there is no clear asymmetry in the probability distribution of $\partial c_{p} / \partial y^{*}$ (see figure $2 c$ ) meaning that the bistable behavior of the flow is altered. This configuration is associated with base pressure recovery and a drag reduction of $5.8 \%$ (see table 1) while an increase in the recirculation length is observed. For the position $\mathrm{V}_{d}$, the perturbation has no significant effect on the flow. The base pressure is equal to the natural value and the two peaks in the probability distribution of $\partial c_{p} / \partial y^{*}$ are recovered: the two states $\# \mathrm{P}$ and \#N coexist. The lift force measured for the four configurations is very close to the natural value (see table 1 ). Thus, the vertical control cylinder does not seem to affect the pressure distributions on the upper and lower sides of the body. 


\begin{tabular}{|c|c|c|c|c|c|c|c|c|}
\hline Pos. & $x_{C}^{*}$ & $z_{C}{ }^{*}$ & $\frac{\partial C_{p}}{\partial z^{*}}$ & $\mathcal{A}=\sqrt{\left\langle{\frac{\partial c_{p}}{\partial y^{*}}}^{2}\right\rangle}$ & $C_{x p}$ & $C_{z p}$ & $C_{p b}$ & $L_{r}{ }^{*}$ \\
\hline \multicolumn{2}{|c|}{ Natural $\ddagger$} & & -0.02 & 0.15 & 0.206 & -0.08 & -0.185 & 1.47 \\
\hline $\mathrm{H}_{a}$ & 0.37 & 1.17 & -0.08 & 0.06 & 0.193 & -0.06 & -0.172 & 1.54 \\
\hline $\mathrm{H}_{b}$ & 0.58 & 0.25 & -0.01 & 0.07 & 0.196 & -0.09 & -0.175 & 1.54 \\
\hline $\mathrm{H}_{c}$ & 0.71 & 0.21 & 0.16 & 0.01 & 0.234 & -0.14 & -0.212 & 0.86 \\
\hline $\mathrm{H}_{d}$ & 1.00 & 0.60 & 0.01 & 0.14 & 0.206 & -0.08 & -0.188 & 1.45 \\
\hline
\end{tabular}

TABLE 2. Global properties of the natural and disturbed wakes for four different configurations of horizontal control cylinder. $\ddagger$ Data from Grandemange et al. $(2013 b)$.

It is now convenient to define an indicator of asymmetry that is independent of the repartition of the two asymmetric states. When the flow is symmetric in average but spontaneously asymmetric because of the bi-stability, the superposition of the two asymmetric states induces important variations of $\partial c_{p} / \partial y^{*}$ : typically for equiprobable states, $\operatorname{Std}\left(\partial c_{p} / \partial y^{*}\right) \approx 0.16$ while $\partial C_{p} / \partial y^{*} \approx 0$. In opposition, when the flow is locked in one of the asymmetric states: $\operatorname{St}\left(\partial c_{p} / \partial y^{*}\right) \approx 0$ while $\partial C_{p} / \partial y^{*} \approx \pm 0.16$. Hence, the root mean square of the gradient, denoted $\mathcal{A}$, is used to quantify the degree of asymmetry:

$$
\mathcal{A}=\sqrt{\left\langle\left(\frac{\partial c_{p}}{\partial y^{*}}\right)^{2}\right.}=\sqrt{\frac{\partial C_{p}^{2}}{\partial y^{*}}+S t d\left(\frac{\partial c_{p}}{\partial y^{*}}\right)^{2}} .
$$

In table 1 , it can be noticed that $\mathcal{A}>0.15$ for the configurations $\mathrm{V}_{a}, \mathrm{~V}_{b}$ and $\mathrm{V}_{d}$ for which the asymmetric states are observed.

In contrast to the vertical cylinder, the horizontal control cylinder always preserves the reflectional symmetry of the set-up. For positions $\mathrm{H}_{a}$ and $\mathrm{H}_{b}$ in figures $3(a, b)$, the cylinder is located in the inner shear layers developing from the upper and lower side of the geometry respectively. First, for $\mathrm{H}_{a}$, the top/bottom organization of the recirculation region is significantly modified in comparison to the natural case $\nmid$ which is consistent with the negative value of $\partial C_{p} / \partial z^{*}$ (see table 2). On the other hand, the flow modifications for $\mathrm{H}_{b}$ are almost undetectable and $\partial C_{p} / \partial z^{*}$ is close to the natural value. However, in both cases, there is an increase in the recirculation lengths and a suppression of the bi-stability. There is only one preferred position centered on 0 visible in the probability distribution of $\partial c_{p} / \partial y^{*}$ with $\mathcal{A} \approx 0.07$. In parallel, it is measured that these positions achieve base pressure recovery and up to $6.3 \%$ reduction of pressure drag.

At the location $\mathrm{H}_{c}$ in figure $3(c)$, the cylinder is in the outer shear layer coming from the lower side of the geometry. It generates a vertical jet by deviating flow from the mixing layer to the center of the recirculation region. The organization of the near wake is strongly modified. A curved jet develops from the control cylinder and reaches the top of the base. It is associated with the large pressure gradient in the $z$ direction: $\partial C_{p} / \partial z^{*}=0.16$. This control cylinder position is a high drag case $\left(C_{p b}=-0.212\right)$ which is ascribed to the strong curvature of the jet and the reduced recirculation length. The probability distribution of $\partial c_{p} / \partial y^{*}$ shows that the wake is clearly stable in the centered state. For the last case presented, the control cylinder is in the middle of the recirculation

$\dagger$ The natural case detailed in Grandemange et al. (2013b) is not presented here; to facilitate comparison, it is worth mentioning that the natural wake has similar features to the one presented in figure $3(d)$. 
Figure 4. Effect of the vertical control cylinder on the pressure on the body: $\partial C_{p} / \partial y^{*}$ at $y^{*}=0,(a) ; C_{y p},(b) ; \mathcal{A},(c) ; C_{p b},(d)$. Contour intervals are $0.03(a), 0.01(b), 0.02(c)$ and 0.005 $(d)$; continuous and dashed line are positive and negative values respectively; contour 0 is not plotted. Thick lines are $U_{x}$ contours of the natural flow: $\cdots, U_{x}{ }^{*}=0.1 ;-, 0.5 ;---, 0.9$.

FIGURE 5. Effect of the horizontal control cylinder on the pressure on the body: $\partial C_{p} / \partial z^{*}$, $(a) ; C_{z p},(b) ; \mathcal{A},(c) ; C_{p b},(d)$. Contour intervals are $0.03(a), 0.02(b), 0.01(c)$ and $0.005(d)$; continuous and dashed line are positive and negative contours respectively; contour 0 is not plotted. Thick lines are $U_{x}$ contours of the natural flow: $\cdots, U_{x}{ }^{*}=0.1 ;-, 0.5 ;---, 0.9$.

region (position $\mathrm{H}_{d}$ ). The bi-stability of the wake is clearly observable and all the global properties listed in table 2 are similar to the natural ones.

\subsection{Sensitivity analysis}

Figure 4 and 5 present the results of the sensitivity analyses obtained for many control cylinder positions in the wake following the methodology described in section 2.2. We first focus on the maps of figure 4 obtained with the vertical control cylinder. Figure $4(a)$ is the sensitivity map of the mean pressure gradient $\partial C_{p} / \partial y^{*}\left(x_{C}^{*}, y_{C}^{*}\right)$ which is a symmetry indicator of the mean recirculation bubble. For $y_{C}{ }^{*} \neq 0$, the control cylinder almost always selects a wake orientation depending on its position in both the $x$ and $y$ directions. However, as soon as the symmetry of the geometry is preserved, i.e. $y_{C}{ }^{*}=0$, the flow retrieves its reflectional symmetry in average with $\partial C_{p} / \partial y^{*} \approx 0$. A second indicator of the flow symmetry is the mean side force which can be estimated from the pressure taps on the lateral sides of the geometry. From these measurements, it is observed that the asymmetric pressure distributions on the base are associated with a force in the $y$ direction (see figure $4 b$ ). In most of the control cylinder positions where $\left|\partial C_{p} / \partial y^{*}\right|>0.1$, a value of $C_{y p} \approx \pm 0.02$ is measured which corresponds to the side force of the asymmetric states $\# \mathrm{P}$ and $\# \mathrm{~N}$ of the natural wake. Wherever $\partial C_{p} / \partial y^{*} \approx 0, C_{y p}$ is equally measured close to 0 which confirms the symmetry of the pressure distribution around the whole geometry.

The sensitivity map $\mathcal{A}\left(x_{C}{ }^{*}, y_{C}{ }^{*}\right)$ is shown in figure $4(c)$. For most of the locations, $\mathcal{A}$ is close to 0.16 . This indicates that either the flow is bistable (for $\partial C_{p} / \partial y^{*} \approx 0$ ) or the asymmetry in the $y$ direction is at least equal to that of states \# $\mathrm{P}$ and $\# \mathrm{~N}$. The exceptions are measured in the center of the recirculation bubble, for $x_{C}{ }^{*} \approx 0.6$ and $y_{C}{ }^{*} \approx 0$. For such positions, the diminution of $\mathcal{A}$ indicates that the bistable behavior is suppressed and like for the configuration $\mathrm{V}_{c}$ (see figure $2 c$ and table 1 ) these cylinder positions achieve base pressure recovery. The sensitivity map of $C_{p b}\left(x_{C}{ }^{*}, y_{C}{ }^{*}\right)$ in figure $4(d)$ shows that $C_{p b}$ increases are concentrated around the center of the recirculation region where the symmetry of the flow is preserved and the bi-stability is suppressed. However, when the lateral mixing layers are disturbed, the base pressure tends to decrease. For the other control cylinder positions, $C_{p b}$ is measured close to the natural value of $C_{p b n}=-0.185$.

The results of the sensitivity analysis obtained with the horizontal cylinder are shown in figure 5. As this perturbation preserves the reflectional symmetry of the set-up, both the mean side force and $\partial C_{p} / \partial y^{*}$ remain nil for all the disturbance positions. Thus, the considered global quantities are the mean vertical pressure gradient $\partial C_{p} / \partial z^{*}$, the degree of asymmetry $\mathcal{A}$, the mean lift coefficient $C_{z p}$ and the base pressure $C_{p b}$.

The sensitivity map $\partial C_{p} / \partial z^{*}\left(z_{C}{ }^{*}, y_{C}{ }^{*}\right)$ in figure $5(a)$ shows the significant modifications of the pressure gradient when the control cylinder is in the shear layers (as 
FiguRE 6. Scatter plots of $C_{y p}$ vs. $\partial C_{p} / \partial y^{*}(a)$ and of $S t d\left(\partial c_{p} / \partial y^{*}\right) v s . \partial C_{p} / \partial y^{*}(b)$ for the vertical control cylinder. The dashed line is the linear fit. Crosses are natural values.

Figure 7. Scatter plots of $\mathcal{A}$ vs. $C_{p b}(a)$ and of $C_{x p}$ vs. $C_{p b}(b)$ for the vertical control cylinder. The dashed line is the linear law $C_{x p}=-C_{p b}+0.018$ (not a fit). Crosses are natural values.

illustrated in figures $3 a, b, c)$. In the lower shear layer, $\partial C_{p} / \partial z^{*}>0$ and in the upper shear layer, $\partial C_{p} / \partial z^{*}<0$. Elsewhere, the gradient is close to 0 like in the natural case. It is worth noting that this map strongly differs from the one presented in figure $4(a)$ due to the absence of bi-stability in the $z$ direction. Figure $5(b)$ presents the lift modifications sensitivity map $C_{z p}\left(z_{C}{ }^{*}, y_{C}{ }^{*}\right)-C_{z p n}, C_{z p n}$ being the natural lift coefficient. The perturbation of the top and bottom mixing layers induces significant variations: the lift is increased with the cylinder in the upper shear while it is reduced in the lower one. The degree of asymmetry $\mathcal{A}\left(z_{C}{ }^{*}, y_{C}{ }^{*}\right)$ is presented in figure $5(c)$. When the cylinder is located in the shear layers, $\mathcal{A}<0.1$ so the bi-stability is suppressed as in figures $4(a, b, c)$. Everywhere else, the measurement of $\mathcal{A} \approx 0.16$ is associated with the cohabitation of the asymmetric states: as for $\mathrm{H}_{d}$ (see figure $4 d$ ), the wake is bistable.

The base pressure in figure $5(d)$ is increased up to $6 \%$ when the control cylinder acts on the inner part of the mixing layers in the near wake. However, the lower mixing layer is particularly sensitive as illustrated by the difference observed between $\mathrm{H}_{b}$ and $\mathrm{H}_{c}$ in figure 3 .

\subsection{Correlations between the modified global quantities of the wake}

Correlations between the global quantities modified by a same disturbance are informative about the flow physics. We first consider the correlation in figures $4(a, b)$ between the base pressure gradient $\partial C_{p} / \partial y^{*}$ and the mean side force $C_{y p}$. The relationship between these two parameters is presented in the scatter plot in figure $6(a)$ : each point corresponds to one position of the vertical cylinder and the data globally follow a linear dependence. It is worth mentioning that, by definition, the evaluation of $C_{y p}$ is performed using exclusively the pressure taps on the sides and on the fore-body whereas $\partial C_{p} / \partial y^{*}$ is defined only from the pressure measurements on the base. As a result, the two quantities rely on independent pressure data. One can conclude that the base pressure gradient in the $y$ direction contains the information of the circulation associated with the side force on the geometry.

In equation (3.1), the degree of asymmetry $\mathcal{A}$ is the sum of two terms containing the mean asymmetry of the base pressure $\partial C_{p} / \partial y^{*}$ and its fluctuations $\operatorname{Std}\left(\partial c_{p} / \partial y^{*}\right)$. The correlations between these mean and fluctuating contributions are presented in figure $6(b)$. Most of the data are observed on the circle centered on 0 of radius $0.16 \pm 0.01$, i.e. for $\mathcal{A} \approx 0.16$. All the configurations on that circle are wakes with different proportions of states \#P and \#N. This scatter plot confirms that $\mathcal{A}$ is a relevant parameter to quantify the asymmetry of the near wake. Now, some data are not located on that circle but at a reduced level of $\mathcal{A}$. These points correspond to the cylinder positions close to $\mathrm{V}_{c}$, in the center of the recirculation region (see figures $2 c$ and $4 c$ ). As mentioned in section 3.2, these points are associated with base pressure recovery and then drag reduction (see figure $4 d$ ).

The scatter plot of $\mathcal{A} v s . C_{p b}$ is given in figure $7(a)$. There is a high density of points close to the natural levels at $\mathcal{A} \approx 0.16$ and $C_{p b} \approx-0.185$. Some data report significant base pressure decrease $\left(C_{p b}<-0.19\right)$ for $\mathcal{A}=0.15 \pm 0.03$ : they are associated with the disturbance of the lateral mixing layers. Nevertheless, for $C_{p b}>-0.19$, it is clear that 
Figure 8. Scatter plots of $C_{z p}$ vs. $\partial C_{p} / \partial z^{*}(a)$ and of $C_{z p}$ vs. $C_{p b}$ (b) for the horizontal cylinder: black, $\mathcal{A}<0.1$; red (bright gray), $\mathcal{A}>0.1$. The dashed line is the linear fit $C_{z p}=-0.083-0.26 \partial C_{p} / \partial y^{*}$. Cross is natural value.

there is a correlation between the two quantities: the optimal base pressure recovery corresponds to the minimum level of asymmetry of the recirculation region.

The present experiments show that the average level of base pressure can be considered as a drag indicator. Indeed, there is a strong dependence between base pressure and pressure drag as shown in the scatter plot in figure $7(b)$. Since the evaluation of the pressure drag uses all the taps on the base so that there is, by construction, a certain correlation between these two quantities. However, the affine dependence between $C_{x p}$ and $C_{p b}$ with a slope -1 proves that the disturbance does not affect the pressure distribution on the fore-body: the total pressure drag is the sum of the contribution of the fore-body which is constant at first order and of the base which depends on the control cylinder position.

Similarly, an affine dependence between $\partial C_{p} / \partial z^{*}$ and $C_{z p}$ is found in figure $8 a$. The data superimpose on the fit

$$
C_{z p}=-0.083-0.26 \frac{\partial C_{p}}{\partial z^{*}} .
$$

In contrast to the dependence between the side force and the pressure gradient in the $y$ direction presented in figure $6(a)$, a negative lift coefficient is obtained for $\partial C_{p} / \partial z^{*}=0$; this point is ascribed to the ground proximity. As a consequence, the pressure gradient in the $z$ direction reproduces the modifications of the lift but the presence of the ground introduces an offset.

The relationship between $C_{x p}$ and $C_{z p}$ is studied using a conditional analysis based on the value of $\mathcal{A}$. In figure 8, degrees of asymmetry with $\mathcal{A}$ larger than 0.1 in red (or bright gray) are associated with bistable flows while the data with $\mathcal{A}<0.1$ in black correspond to flows without bi-stability. Figure $8(b)$ shows that the bistable data are concentrated around the natural point. In contrast, the stable data are measured over a wide range of base pressures $\left(C_{p b} \in[-0.22,-0.17]\right)$ presenting a parabolic-like dependence towards the lift coefficient. The positions of base pressure recovery are associated with $C_{z p}$ close to the natural value whereas the base pressure decreases are reported for significant lift modifications and then important recirculation asymmetries in the $z$ direction (large values of $\left.\left|\partial C_{p} / \partial z^{*}\right|\right)$. These latter cases often correspond to the creation of a curved jet into the recirculation region as visible in figure $3(c)$. More generally, the scatter plot in figure $8(b)$ confirms that the domain of drag reduction associated with the horizontal control cylinder is limited and only correspond to few precise configurations.

\section{Discussion}

After presenting the probable mechanisms leading to the suppression of the bi-stability in section 4.1, the dependencies between the drag and the cross-flow forces are analyzed in section 4.2. Then, section 4.3 quantifies the drag ascribed to the bi-stability and discusses more generally the suppression of the symmetry breaking modes as a control strategy for drag reduction of three-dimensional bluff bodies.

\subsection{On the suppression of the bi-stability}

In section 3, it is found that the natural bistable behavior of the wake is suppressed by the control cylinders in two different cases: 
Figure 9. $(a, b)$ Domains of vertical control cylinder positioned in the sensitivity maps of $C_{y p}$ and $\mathcal{A}:-, \mathrm{D}_{1} ;---, \mathrm{D}_{2} .\left(\right.$ c) $C_{y p}$ vs. $C_{x p}$ for vertical control cylinder positions in the domains $\mathrm{D}_{1}$ (red or bright gray) and $\mathrm{D}_{2}$ (black); $\times$, natural values of the asymmetric states; - -, $2^{\text {nd }}$ order polynomial fit.

- for the vertical cylinder in the center of the recirculation, i.e. near the case $\mathrm{V}_{c}$ (figure 2c);

- for the horizontal cylinder in the top and bottom mixing layers like the configurations $\mathrm{H}_{a}, \mathrm{H}_{b}$ and $\mathrm{H}_{c}$ (figure $3 a, b, c$ ).

The mechanisms responsible for the suppression of the bi-stability for these configurations may be detailed from the results of Grandemange et al. (2013a) who investigated the condition of existence of bi-stability through a parametric study of both the base body aspect ratio and the ground clearance height. Indeed, this experimental work evidences that the $y$ instability (responsible for the bi-stability) corresponds to antisymmetric perturbations in the $y$ directions and to symmetric ones in the $z$ directions. From this statement, one can interpret the vertical cylinder in the plane $y^{*}=0$ as a symmetric forcing of the flow, maybe due to the boundary condition imposing $u_{y}{ }^{*}=0$ on its surface. However, from figure $4(c)$, it is clear that the sensitivity of the flow is not uniform in the plane $y^{*}=0$ : for example, forcing the symmetry at the end of the recirculation bubble is useless (see configuration $\mathrm{V}_{d}$ in figure $2 d$ ).

On the other hand, the horizontal cylinder in the top and bottom shear layers is likely to introduce an asymmetry in the $z$ direction, preventing the development of the $y$ instability. This interpretation is confirmed by the comparison of figures $3(a, c)$ to figure $3(d)$. As a result, in these cases, the suppression of the bi-stability seems associated with a breaking of the relative top/bottom symmetry of the recirculation bubble observed in the natural wake.

\subsection{On the drag of the disturbed wakes}

The dependence between the drag and the cross-flow forces are now considered. We first discuss the lateral force and then the lift force before providing a synthesis on the drag contributions.

\subsubsection{Drag due to cross-flow forces}

In section 3.1, it is shown that the vertical disturbance generates modifications of drag and side force while the modifications of lift are negligible. Thus, the data obtained using the vertical disturbance are relevant to study the dependencies between the side force and the drag. To do so, two different domains of disturbance positions are considered:

- the domain $\mathrm{D}_{1}$ for $\left(x_{C}{ }^{*}, y_{C}{ }^{*}\right) \in[1.08,1.46] \times[-0.21,0.21]$;

- the domain $\mathrm{D}_{2}$ for $\left(x_{C}{ }^{*}, y_{C}{ }^{*}\right) \in[0.70,0.87] \times[-0.49,0.49]$.

These two domains are presented in figures $9(a, b)$ which are parts of the sensitivity maps of $C_{y p}$ and $\mathcal{A}$ (extracted from figures $\left.4 b, c\right)$. In both domains, when the disturbance goes from the negative to the positive values of $y_{C}{ }^{*}$, the body undergoes a mean side force from of about -0.025 to +0.025 which is imputed to state \#P and \# $\mathrm{N}$ respectively. The difference between both domains lies in the degree of asymmetry $\mathcal{A}$ for $y_{C}{ }^{*} \approx 0$. In the domain $\mathrm{D}_{1}, \mathcal{A}$ is constant at first order; the position $y_{C}{ }^{*}$ of the cylinder selects the proportion of the states \#P and \#N. The equiprobability is obtained for $y_{C}{ }^{*}=0$ so that the wake is clearly bistable for $y_{C}{ }^{*} \approx 0$ (like $\mathrm{V}_{d}$ in figure $2 d$ ). In the domain $\mathrm{D}_{2}$, the degree of asymmetry $\mathcal{A}$ diminishes continuously as the mean lateral force tends to 0 and the wake bi-stability is suppressed for $y_{C}{ }^{*} \approx 0$ (like $\mathrm{V}_{c}$ in figure $2 c$ ). 
Figure 10. Scatter plot of $C_{x p} v s .\left\langle c_{y p}{ }^{2}\right\rangle$ for vertical control cylinder positions in the domains $\mathrm{D}_{1}$ (red or bright gray) and $\mathrm{D}_{2}$ (black);---, affine fit.

The correlation between $C_{y p}$ and $C_{x p}$ is revealed in the scatter plot in figure $9(c)$. Depending on the domain $\left(\mathrm{D}_{1}\right.$ or $\left.\mathrm{D}_{2}\right)$, two different drag vs. side force behaviors are observed. First, in the domain $\mathrm{D}_{1}$ (red or bright gray dots), the drag is measured constant at $C_{x p}=C_{x p n} \pm 0.002$ and independent of the side force in the whole range of $C_{y p}$. On the other hand, the drag from the domain $\mathrm{D}_{2}$ (black dots) depends on the side force; the minimum of drag is measured for $C_{y p} \approx 0$ and progressively increases with $\left|C_{y p}\right|$ leading to a parabolic dependence. As a result, an additional drag proportional to $C_{y p}{ }^{2}$ seems ascribed to the side force for control cylinders in the domain $\mathrm{D}_{2}$; this drag contribution is denoted by $C_{x p}^{y}$.

The fact that such a quadratic dependence is not observed in the domain $\mathrm{D}_{1}$ can be explained as follows. When located in the domain $\mathrm{D}_{1}$, the disturbance gradually modifies the repartition between the states \#P and \#N so that all the values of the lateral force between -0.025 and 0.025 are explored. However, the side force is always close to the level of the current asymmetric states, i.e. $\left|c_{y p}\right| \approx 0.025$. Thus, if the presence of a side force generates an additional drag, an alternating side force even nil in average must equally produce additional drag.

The mean square of the lateral force $\left\langle c_{y p}{ }^{2}\right\rangle$ measures both the steady and unsteady contribution in the $y$ direction. The scatter plot in figure 10 shows a linear correlation between $C_{x p}$ and $\left\langle c_{y p}{ }^{2}\right\rangle$, confirming the previous analysis. Indeed, the data associated with both the domain $\mathrm{D}_{1}$ and $\mathrm{D}_{2}$ now follow the same trend that can be fitted by the affine law:

$$
C_{x p}=20\left\langle c_{y p}\right\rangle+0.18 .
$$

Consequently, the quantification of the total pressure drag $C_{x p}$ contains an additional contribution of the side force denoted $C_{x p}^{y}$ which must take into account both the mean side force and its fluctuations:

$$
C_{x p}-C_{x p 1}=C_{x p}^{y}=a_{y}\left\langle c_{y p}^{2}\right\rangle=a_{y}\left[C_{y p}^{2}+S t d\left(c_{y p}\right)^{2}\right],
$$

with $a_{y} \approx 20 . C_{x p 1}$ is the pressure drag that can be achieved for a steady nil side force.

At this stage, it is important to mention that the measurement of $\left\langle c_{y p}{ }^{2}\right\rangle$ suffers from a lack of accuracy partially because the statistics are not fully converged. This is visible through the dispersion of the data along the vertical axis in figure 10 . The value of $\left\langle c_{y p}{ }^{2}\right\rangle$ tends to be overestimated, independently of $C_{x p}$. Thus, a non-negligible part of $\left\langle c_{y p}{ }^{2}\right\rangle$ is not responsible for induced drag; in other words, $C_{x p 1}$ in equation (4.2) can not be evaluated at 0.18 as it would be expected from the fit (4.1). However, this does not affect the following analyses since only the slope of the affine fit (4.1) is used.

The drag vs. lift dependence is now considered when the wake is disturbed by the horizontal control cylinder (data plotted in figure 8). However, there are not only evolutions of lift but also of side force since there are stable and bistable cases. To get rid of the additional drag introduced by the fluctuations of side force and to obtain the drag evolutions ascribed to the lift only, one may subtract the term $C_{x p}^{y}=20\left\langle c_{y p}{ }^{2}\right\rangle$. Even if this term certainly overestimates the drag ascribed to the side force, it should not alter the drag vs. lift dependence: the important point is that the overestimation is independent of $C_{z p}$.

In figure 11 are plotted the dependence between $\left(C_{x p}-C_{x p}^{y}\right)$ and $C_{z p}$. Unlike the scatter plot in figure $8(b)$, the data now present a global coherence: both the stable and bistable data superimpose on the same master curve. The dependence can be approximated by 
Figure 11. Dependence between $C_{x p}-C_{x p}^{y}$ and $c_{z p}$ for horizontal control cylinder positions $\left(x_{C}{ }^{*}, z_{C}{ }^{*}\right) \in[0.21,1.67] \times[0.15,1.30]$ sorted on the value of $\mathcal{A}$ : red (bright gray), $\mathcal{A}>0.10 ;$ black, $\mathcal{A}<0.10 ;---, 2^{\text {nd }}$ order polynomial fit.

the $2^{\text {nd }}$ order polynomial fit

$$
C_{x p}-C_{x p}^{y}=C_{x p 0}+C_{x p}^{z}
$$

with

$$
C_{x p}^{z}=a_{z}\left(C_{z p}-C_{z p 0}\right)^{2}
$$

and $a_{z}=9.9, C_{z p 0}=-0.079$. As a result, $C_{x p 0}$ corresponds to the minimal value of drag that can be achieved for a stable wake with optimal cross-flow forces but its accurate evaluation is impossible using the fit (4.3) due to the overestimation of $C_{x p}^{y}$. In parallel, it is observed that $C_{z p 0} \neq 0$ : the optimal drag point does not correspond to the minimum of $C_{z p}{ }^{2}$ contrary to what is found for the force in the $y$ direction. It seems rather to be related to the best homogeneity of the base pressure distribution: $\partial C_{p} / \partial z^{*} \approx 0$ for $C_{z p 0} \approx-0.08$.

Now that the influence of each of the cross-flow forces on pressure drag are characterized, a synthesis is presented next with a confrontation to notions of induced drag for lifting airfoils.

\subsubsection{Synthesis on the drag contributions}

The quadratic dependencies between drag and side forces presented above are reminiscent of the notions of induced drag commonly used in aeronautics (Batchelor 2002). For an airfoil of lift coefficient $C_{L}$, the total drag coefficient $C_{D}$ is usually separated into a parasitic drag and an induced drag (which is part of the pressure drag) that relies on three-dimensional effects. This term of induced drag is associated with the lift which can also be ascribed to the pair of counter-rotating vortices downstream of the wingtips. Denoting $C_{D 0}$ and $C_{D i}$ the coefficients associated with the parasitic and induced drag respectively, the total drag coefficient verifies:

$$
C_{D}=C_{D 0}+C_{D i}
$$

with

$$
C_{D i}=\alpha_{L} C_{L}^{2}
$$

and $\alpha_{L}$ depending on the geometry of the wing and more precisely, on the force distribution along the span.

In the framework of car aerodynamics, the results of Ahmed et al. (1984) demonstrates the critical impact of the three-dimensional flow structures on the drag. For after-body slant angles $\phi \in\left[13^{\circ}, 25^{\circ}\right]$, an increase of drag associated with the development of an intense pair of counter-rotating vortices is reported. Exploring the effect of flaps mounted on the after-body in the $25^{\circ}$ case, Beaudoin \& Aider (2008) prove the strong correlation between the drag, the lift and the pair of counter-rotating vortices. Moreover, extending the use of flaps at the trailing edge of a square-back configuration, Grandemange et al. $(2013 c)$ confirm that induced drag phenomena are relevant to interpret the drag evolutions ascribed to the modification of the flap angles.

The theory of induced drag in relation (4.6) can be extended to the analysis of the aerodynamic forces in a road vehicle configuration considering the lift force but also the side force. Moreover, both the steady and unsteady side forces must be taken into account due to the bistable behavior. Now, there is no apparent reason for considering the fluctuations of forces from bi-stability only. Even if it could not be evidenced in 
this work, the fluctuations of cross-flow forces related to oscillating global modes and to turbulent activity (at least at large scales) may logically be added, in both the $y$ and $z$ directions. As a consequence, the following expression for the pressure drag is suggested:

$$
C_{x p}=C_{x p 0}+C_{x p i}
$$

with

$$
C_{x p i}=\alpha_{y}\left\langle c_{y p}^{2}\right\rangle+\alpha_{z}\left\langle\left(c_{z p}-C_{z p 0}\right)^{2}\right\rangle .
$$

This expression (4.8) relies on three parameters: $\alpha_{y}, \alpha_{z}$ and $C_{z p 0}$. As previously mentioned, $C_{z p 0}$ is certainly an offset due to the presence of the ground and hence it is likely to rely on the precise configuration of the vehicle such as the ground clearance. However, this optimal lift correspond to the best homogeneity of the base pressure in the $z$ direction (see figure $8 a$ ) which may be a general rule. By extending the interpretation of $\alpha_{L}$ in aeronautics, one would expect the coefficients $\alpha_{y}$ and $\alpha_{z}$ to depend on the force distribution in the $y$ and $z$ directions. In the present experiment, $\alpha_{y}$ is thought to be close to $a_{y}$ because $a_{y}=20$ is obtained in equation (4.1) from the sensitivity analysis in the domains $\mathrm{D}_{1}$ and $\mathrm{D}_{2}$ only. There, the cylinder is out of the lateral shear layers and it only controls the natural circulation around the geometry. So $C_{x p}^{y}=20\left\langle c_{y p}{ }^{2}\right\rangle$ is the drag induced by the natural lateral fluctuations of the wake, including bi-stability, independently of the control cylinder. Differently, it is clear that the horizontal cylinder produces a lift on the body, especially when it is located in the mixing layers. Consequently, the measurement of $a_{z}$ in equation (4.4) is associated with the system comprised of the main geometry and the disturbance while $\alpha_{z}$ is related to the main geometry only. In other words, one expects $\alpha_{z} \neq a_{z}$.

\subsection{On the stabilization of the symmetric state as a control strategy for drag reduction}

An estimation of the additional drag due to the bi-stability of the natural wake can be provided by the sensitivity analysis. $\left\langle c_{y p}{ }^{2}\right\rangle$ is measured at $9.010^{-4}, 8.810^{-4}$ and $8.910^{-4}$ for the states $\# \mathrm{P}, \# \mathrm{~N}$ and the mean symmetric flow respectively; these values give an average of $C_{x p}^{y}=0.018$. However, the direct application of equation (4.1) with $C_{x p}^{y}=20\left\langle c_{y p}{ }^{2}\right\rangle$ may over-estimate the contribution of the bi-stability so $C_{x p}^{y}<0.018$. Now, an inferior limit can be obtained from the natural flow. Using a conditional averaging technique (Grandemange et al. 2013b), the side force is measured at $\left|C_{y p}\right|=0.021$ for both mirror states. Hence, it is obtained that $C_{x p}^{y}>20 C_{y p}{ }^{2} \approx 0.008$ and this inferior bound is free from the over-estimation of $\left\langle c_{y p}{ }^{2}\right\rangle$. As a conclusion, the drag related to the bi-stability is estimated between 0.008 and 0.018 , i.e. between $4 \%$ and $9 \%$ of the total pressure drag.

In Grandemange et al. (2012a), it is found that the origin of the bi-stability is ascribed to a pitchfork bifurcation of the laminar wake. For Re $>340$, the steady symmetric flow disappears and the wake solutions are two mirror steady asymmetric states. At large Reynolds numbers, turbulent fluctuations allow the exploration of the two solutions but the symmetric flow is never observable in a strict sense, at least up to $\operatorname{Re} \sim 10^{7}$ (Grandemange et al. 2013b). The effect of the vertical cylinder placed at the center of the recirculating bubble (see figure $2 c$ ) might be interpreted as a control of the reminiscences of the steady reflectional symmetry breaking mode, which renders the symmetry of the wake.

Now, steady pitchfork bifurcations are common feature of three-dimensional flows; for instance Pier (2008) evidences that for a sphere, the loss of axisymmetry is a source of additional drag. Hence, the stabilization of this steady symmetric regime offers a 
relevant control strategy for drag reduction of three-dimensional bluff bodies, even at large Reynolds numbers.

\section{Concluding remarks}

Using horizontal and vertical control cylinders as perturbations, the sensitivity analyses of the separated flow past a simplified ground vehicle reveal the receptive zones of the wake. First, a disturbance that breaks the reflectional symmetry of the set-up selects one of the two asymmetric states depending on both the streamwise and lateral positions of the perturbation. Besides, the receptive region of the bi-stability to a steady symmetric forcing is found in the center of the recirculation region. These positions of vertical cylinder lead to a stable centered wake with an increased recirculation length and a base pressure recovery. Moreover, the sensitivity maps obtained with a vertical control cylinder show strong similitude with the ones presented in Grandemange et al. (2012b) for the separated flow past an axisymmetric trailing edge. Thus, the bi-stability of these two geometries is very likely to result from the same physics. Given the generality of these bistable phenomena, the high receptivity of the center of the recirculation to a symmetric forcing may be a widespread characteristic of three-dimensional separated flows.

The sensitivity maps obtained with the horizontal control cylinder indicate that the receptivity is concentrated in the upper and lower shear layers. Significant lift modification are reported, especially when the control cylinder generates a jet from the high momentum flow to the recirculation region. Such a wake asymmetry in the $z$ direction is likely to prevent the development of the $y$ instability responsible for the bi-stability which results, in some cases, in drag reduction.

From these complementary sensitivity analyses, correlations between the drag and the cross-flow forces are identified. Extending the formalism induced drag in aeronautics, a quantification of the impact of the cross-flow forces on the pressure drag is suggested. It is found that both the steady and unsteady cross-flow forces must be taken into account. The optimal point in terms of induced drag is obtained for a steady force at $C_{y p}=0$ and $C_{z p}=-0.08$. The fact that the optimal lift is negative should be ascribed to the ground proximity and may differ from one experiment to another; however, these forces correspond to the best uniformity in the base pressure distribution which is very likely to be a general result.

As a consequence, it is confirmed that the bi-stability of the natural flow is responsible for part of the drag, estimated between $4 \%$ to $9 \%$ of the total pressure drag. This evaluation quantifies the potential of drag reduction on the Ahmed geometry by using control devices able to force a centered wake that is steady at long time-scales.

More generally, one can wonder if the drag dependence on the fluctuations of cross-flow forces stands for non-bistable wakes at high Reynolds number. This question may motivate future studies which could lead to the definition of new strategies of drag reduction.

\section{Acknowledgments}

The authors wish to thank G. Pujals from PSA Peugeot Citroën for productive discussions on the analysis of these experimental results. 
Ahmed, S.R., Ramm, G. \& Faitin, G. 1984 Some salient features of the time-averaged ground vehicle wake. SAE Technical Paper Series $\mathbf{8 4 0 3 0 0 .}$

BAtChelor, G.K. 2002 An introduction to fluid dynamics. Cambridge University Press.

Beaudoin, J.F. \& Aider, J.L. 2008 Drag and lift reduction of a three-dimensional bluff body using flaps. Experiments in Fluids 44 (4), 491-501.

Berger, E., Scholz, D. \& Schumm, M. 1990 Coherent vortex structures in the wake of a sphere and a circular disk at rest and under forced vibrations. Journal of Fluids and Structures 4 (3), 231-257.

Fabre, D., Auguste, F. \& Magnaudet, J. 2008 Bifurcations and symmetry breaking in the wake of axisymmetric bodies. Physics of Fluids 20, 051702.

Grandemange, M., Gohlke, M. \& Cadot, O. $2012 a$ Reflectional symmetry breaking of the separated flow over three-dimensional bluff bodies. Physical Review E 86, 035302.

Grandemange, M., Gohlke, M. \& Cadot, O. $2013 a$ Bi-stability in the turbulent wake past parallelepiped bodies with various aspect ratios and wall effects. Physics of Fluids 25, 095103.

Grandemange, M., Gohlke, M. \& Cadot, O. $2013 b$ Turbulent wake past a three-dimensional blunt body. Part 1. Global modes and bi-stability. Journal of Fluid Mechanics 722, 51-84.

Grandemange, M., Mary, A., Gohlke, M. \& Cadot, O. $2013 c$ Effect on drag of the flow orientation at the base separation of a simplified blunt road vehicle. Experiments in fluids $54(5), 1-10$.

Grandemange, M., Parezanović, V., Gohlke, M. \& Cadot, O. $2012 b$ On experimental sensitivity analysis of the turbulent wake from an axisymmetric blunt trailing edge. Physics of Fluids 24, 035106.

Herry, B.B., Keirsbulck, L. \& Paquet, J.B. 2011 Flow bi-stability downstream of threedimensional double backward facing steps at zero-degree slideslip. ASME Transactions Journal of Fluids Engineering 133 (054501), 1-4.

Higuchi, H. 2005 Passive and active controls of three-dimensional wake of bluff body. JSME International Journal Series B $\mathbf{4 8}$ (2), 322-327.

HiLL, D.C. 1992 A theoretical approach for analyzing the restabilization of wakes. NASA Technical Report 103858.

LaWson, N.J., Garry, K.P. \& FAUCOMPRET, N. 2007 An investigation of the flow characteristics in the bootdeck region of a scale model notchback saloon vehicle. Proceedings of the Institution of Mechanical Engineers - part D - Journal of Automobile Engineering 221 (D6), 739-754.

LeE, J.Y., PAik, B.G. \& LeE, S.J. 2009 PIV measurements of hull wake behind a container ship model with varying loading condition. Ocean Engineering 36 (5), 377-385.

Luchini, P., Giannetti, F. \& Pralits, J. 2009 Structural sensitivity of the finite-amplitude vortex shedding behind a circular cylinder. In IUTAM Symposium on Unsteady Separated Flows and their Control, pp. 151-160. Springer.

Magarvey, R.H. \& Bishop, R.L. 1961 Transition ranges for three-dimensional wakes. Canadian Journal of Physics 39 (10), 1418-1422.

Marquet, O., Sipp, D. \& Jacquin, L. 2008 Sensitivity analysis and passive control of cylinder flow. Journal of Fluid Mechanics 615 (-1), 221-252.

Meliga, P., Chomaz, J. \& Sipp, D. 2009a Global mode interaction and pattern selection in the wake of a disk: a weakly nonlinear expansion. Journal of Fluid Mechanics 633, 159-189.

Meliga, P., Сhomaz, J.M. \& Sipp, D. $2009 b$ Unsteadiness in the wake of disks and spheres: instability, receptivity and control using direct and adjoint global stability analyses. Journal of Fluids and Structures 25 (4), 601-616.

Meliga, P., Pujals, G. \& Serre, E. 2012 Sensitivity of two-dimensional turbulent flow past a D-shaped cylinder using global stability. Physics of Fluids 24 (6), 061701-061701.

Morrison, J.F. \& Qubain, A. 2009 Control of an axisymmetric turbulent wake by a pulsed jet. Advances in Turbulence XII pp. 225-228.

Ormières, D. \& Provansal, M. 1999 Transition to turbulence in the wake of a sphere. Physical Review Letters 83 (1), 80-83.

Parezanović, V. \& CADOT, O. 2009 The impact of a local perturbation on global properties of a turbulent wake. Physics of Fluids 21, 071701. 
Parezanović, V. \& CAdOT, O. 2012 Experimental sensitivity analysis of the global properties of a two-dimensional turbulent wake. Journal of Fluid Mechanics 693, 115-149.

PIER, B. 2008 Local and global instabilities in the wake of a sphere. Journal of Fluid Mechanics 603, 39-61.

Sakamoto, H. \& Haniu, H. 1990 A study on vortex shedding from spheres in a uniform flow. Journal of Fluids Engineering 112, 386-392.

Sevilla, A. \& Martinez-Bazan, C. 2004 Vortex shedding in high Reynolds number axisymmetric bluff body wakes: local linear instability and global bleed control. Physics of Fluids 16, 3460 .

Siegel, S., Seidel, J., Cohen, K., Aradag, S. \& Mclaughlin, T. 2008 Open loop transient forcing of an axisymmetric bluff body wake. 46th AIAA Aerospace Sciences Meeting and Exhibit (2008-595).

Strykowski, P.J. \& Sreenivasan, K.R. 1990 On the formation and suppression of vortex shedding at low Reynolds numbers. Journal of Fluid Mechanics 218, 71-107.

Szaltys, P., Chrust, M., Przadka, A., Goujon-Durand, S., Tuckerman, L.S. \& WesFREID, J.E. 2012 Nonlinear evolution of instabilities behind spheres and disks. Journal of Fluids and Structures 28, 483-487.

Wassen, E., Eichinger, S. \& Thiele, F. 2010 Simulation of active drag reduction for a squareback vehicle. Active Flow Control II pp. 241-255.

Weickgenannt, A. \& Monkewitz, P.A. 2000 Control of vortex shedding in an axisymmetric bluff body wake. European Journal of Mechanics-B/Fluids 19 (5), 789-812. 